\title{
The Use of Semi-Automated Method for Assessing the Horizontal Positional Accuracy of Google Earth imagery
}

\author{
Sarhat M Adam, Abdulrahman Farsat Heeto \\ College Engineering, University of Duhok, Duhok, Kurdistan Region - Iraq
}

\begin{abstract}
Google Earth imagery is frequently used in science, engineering, and other mapping applications. However, the company owning the tool announced that the data available in its geographical products is only approximate, so its accuracy is not officially documented. The Google Earth imagery in many areas around the world has been independently checked by scholars and third body parties. The estimated accuracies are found to largely vary depending on various factors but mainly due to, the imagery source or the image resolution. Positional accuracy testing methodology may also affect the assessment results. In processing, there should be many points around the tested area in order for the comparison to be more reliable. In this paper, the horizontal accuracy assessment was carried on the Google Earth imagery in Duhok city using the traces collected via GPS in Real Time Kinematic (RTK) technique. About $38 \mathrm{~km}$ of trajectory was collected for the two main roads in the selected area. Via semi-automated method, the points from RTK trajectory were compared to the corresponding extracted points from the centerline of the road network of Google Earth imagery. The nearest neighboring method through buildup algorithm was considered for comparison between both sets of data. Root Mean Square Error (RMSE) and maximum error were computed for horizontal positional coordinates and found to be $1.53 \mathrm{~m}$ and $7.76 \mathrm{~m}$, respectively.
\end{abstract}

KEYWORDS : parallel processing, neighborhood search, agriculture.

\section{INTRODUCTION}

Google Earth is the most common source of information used in the internet service which offers free access to the worldwide collection of Geo-referenced satellite imagery. This system becomes a significant source of data to the broad spectrum of users such as earth profile, ground coordinates and highway networks which are some of the system benefits among many. Professional users, such as planners and engineers, need to know the level of accuracy that the Google Earth can provide as well as to what extent the positions of such application is dependable. Images are achieved from satellite imagery, aerial photography and GIS 3D globe. Furthermore, Google Earth uses digital elevation model (DEM) data accumulated by NASA's Shuttle Radar Topography Mission (SRTM). The majority of

Academic Journal of Nawroz University

(AJNU) Volume 7, No 4 (2018).

Regular research paper: Published 21 December 2018

Corresponding author's e-mail : sarhat@uod.ac

Copyright (C2017 Sarhat M Adam, Abdulrahman Farsat

Heeto. This is an open access article distributed under the

Creative Commons Attribution License. the high- resolution imagery in Google Earth map is acquired using the Digital Globe Quickbird Satellite sensor. The sensor has a capability to offer $65 \mathrm{~cm}$ pixel and about $2.6 \mathrm{~m}$ multispectral (MS) resolutions at truly nadir view. Google is readily substituting this base imagery with $2.5 \mathrm{~m}$ SPOT images and several higher resolution datasets from the National Imagery Interpretability Rating Scale (NIIRS, 1996, Leachtenauer et al., 1997) Positional accuracy of Google Earth cannot be expected to meet most of the engineering standards. However, for most preliminary projects such as best route selection and rough volume estimation can be beneficial. Highway designers can utilize Google imagery in the early stages of route selection when high spatial coordinate accuracy is not a critical issue; thus, during site selection of a large project, the program can make a useful guide to visualize ground topography regardless of the sub-meters accuracy. The positional accuracy of Google Earth imagery has been investigated and assessed by many researchers. In a work by Potere (2008), he assessed the horizontal positional accuracy and quality of the captured Google Erath imagery. The study selected an average of four points in 109 cities and compared Google Earth with Landsat GeoCover 
imagery accuracy. The overall accuracy, Root Mean Square Error (RMSE) found to be $39.7 \mathrm{~m}$ with a range of 0.4 to 171.6 meters. Different accuracies were found in various world locations. Ubukawa (2013), tested the horizontal positional accuracies of five geospatial datasets of different scales in comparison with ALOS (Advanced Land Observing Satellite) sensor ,The Panchromatic Remote-sensing Instrument for Stereo Mapping (PRISM) imagery which is a panchromatic radiometer has a $2.5 \mathrm{~m}$ spatial resolution at nadir. These imagery which has a higher spatial resolution than Landsat GeoCover studied by (Potere, 2008), and also higher expected positional accuracy. The RMSEs of 6.1 $\mathrm{m}$ were estimated of absolute positional accuracy at nadir for such images (Ubukawa, 2013). Parades and his colleagues worked in 2013 to assess the horizontal positional accuracy of Google Earth by comparing coordinates extracted from a rural cadastral database against coordinates extracted from a well-defined and inferred checkpoints in Google Earth imagery. A standard deviation in the range of 2.3 to $2.9 \mathrm{~m}$, separately for subset of control points over area of medium and high resolution of different sources of images in Google Earth imagery were found with a range of $-16 \mathrm{~m}$ minimum to $20 . \mathrm{m}$ maximum in horizontal coordinates (Paredes-Hernandez et al., 2013). Kazimierz et al. (2011), verified the Google Earth positional accuracy with over 2000 control points located in five continents all over the globe. Their study yields the conclusion that the error had a potential capability of $1.5 \mathrm{~km}$ in some cases such as navigation or education purposes, at certain conditions where its accuracy issues are not considered. Nagi Zomrawi Mohammed et al. (2013), studied the Google Earth positional accuracy of 16 control points situated in Khartoum state, Sudan. The task accomplished by relating the Google Earth measured coordinates of control points with Global Positional System (GPS). The Root Mean Square Errors (RMSE) resulted in $1.59 \mathrm{~m}$ and $1.7 \mathrm{~m}$ respectively for horizontal component and height. On the other hand, Farah and Algarni (2014) investigated the horizontal and vertical accuracy of Google Erath in Riyadh, King Saud University campus by comparing Google Earth imagery estimated coordinates over nine stations coordinates measured by relative positioning in static mode. The computed RMSE for the horizontal position was found to be 2.18 $\mathrm{m}$. While the vertical RMSE of such system was tested on a $700 \mathrm{~m}$ track located outside the University campus. The test divided into 15 stations at a range of $50 \mathrm{~m}$ separation. The RMSE of orthometric height was found to be $1.51 \mathrm{~m}$. (Ragheb and Ragab, 2015), tested part of area and landscape of Ain Shams University Campus, Cairo. Their work evaluated the positional accuracy of Google Earth, comparing coordinates extracted from
Geo-referencing with a base ground survey map. Also, Google Earth imagery used the ERDAS IMAGINE 8.4 software against measured coordinates of points with a Trimble R3, GPS. A horizontal coordinate's deviation was estimated to be in the range of $5.89 \mathrm{~m}$ and $15.68 \mathrm{~m}$, with the RMSE found to be $10.58 \mathrm{~m}$. The recent study on positional accuracy of the Google Earth imagery has been conducted by Maher and Mohammed in the Gaza Strip, Khan Younis city, 2017. A sample of 40 checkpoints distributed in the City in order to compare Google Earth measured coordinates with their corresponding GPS measured coordinates. Low accuracy of horizontal position was achieved in such city, and the authors recommend that the Google Earth imagery was not powerful in their tested area which is mainly due to the low resolution of Google Earth imagery in Gaza strip (El-Hallaq and Hamad, 2017). On the same year, 2017, Goudarzi and Landry tested the horizontal positional accuracy of Google Earth in the city of Montreal, Canada, precise coordinates of 10 GPS points were spatially distributed in the city for comparison with the same points in Google Earth imagery. The results found that the positional accuracy varies in the tested area between $0.1 \mathrm{~m}$ in the South to around 2.7 meters in the North. The previous methods which were used in the positional accuracy assessment of Google Earth imagery are manual and were a point to point comparison. A few researchers considered using the parameters of interpolation or transformation between both data sets as well as in manual sense. To overcome the above drawbacks, an algorithm was developed by the authors using Matlab function to automatically compute RMSEs of the horizontal positional accuracy of Google Earth imagery. The algorithm was used to compare between both the RTK GPS trajectory and Google Earth imagery of around $38 \mathrm{~km} \mathrm{~m}$ within the Duhok city. This algorithm is suitable for evaluating the positional accuracy of the Google Earth imagery or any other imagery sources anywhere in the world. In addition, this technique can solve the drawback of the traditional methods in term of time and a number of collected points in any area of interest.

\section{Data and Methods}

\subsection{Data of experiment}

The image used in this study is the Google Earth imagery database. The company owning the tool employs various imagery sources as previously explained in introductory section. Therefore, the ground sample distance (GSD) of Google Earth imagery is not uniform and areas of high, medium and low resolution were exist in the same location. The supplier of most of the high resolution imagery (pixel size of less than $1 \mathrm{~m}$ ) for Google Earth is Digital Globe. For the selected area of this research, table 1 shows some 
specifications of the related imagery sensors that Google exploited. The work in this paper was implemented to automatically evaluate the positional accuracy of any imagery sources with reference to the RTK technique. Dual frequency GPS receiver was used to collect data for the centerline of the two main roads in the tested area (Duhok city). Around $38 \mathrm{~km}$ of the trajectory with a total number of 2884 points were collected. During the surveying campaign and due to urban nature, the RTK experienced a disconnection issue. As a result, some data was not recorded by the sensor as can be seen in the Figure 1. This was mainly due to the signals being obstructed by the high buildings within the tested area. For the same pathway of RTK, the centerline of the main two roads was carefully drawn on the image using the Google Earth tool. The extraction of the road centerline was carried out with significant consideration.

Table (1) : shows some details about the tested imagery in the research case study (Missions, 2018)

\begin{tabular}{|c|c|c|c|c|c|c|}
\hline $\begin{array}{c}\text { Image } \\
\text { Data }\end{array}$ & $\begin{array}{c}\text { Image } \\
\text { Resolution } \\
\text { (GSD) }\end{array}$ & Source & Company & $\begin{array}{c}\text { Date of } \\
\text { image } \\
\text { taken }\end{array}$ & $\begin{array}{c}\text { Off Nadir } \\
\text { angle of } \\
\text { image }\end{array}$ & Height of Sensor \\
\hline $\begin{array}{c}\text { Google } \\
\text { Earth }\end{array}$ & $\begin{array}{c}0.55 \mathrm{~cm} \text { Pan, } \\
1.84 \mathrm{~m} \mathrm{MS} \text { at } \\
\text { nadir }\end{array}$ & $\begin{array}{c}\text { Digital } \\
\text { Globe }\end{array}$ & $\begin{array}{c}\text { QuickBrid } \\
\text { WorldView- } 1 \\
\text { and } 2\end{array}$ & 2010 & $\begin{array}{c} \pm 40^{\circ} \text { about } \\
\text { nadir }\end{array}$ & $\begin{array}{c}\text { QuickBird:450km } \\
w v 1: 1355 \mathrm{~km} \\
w v 2: 770 \mathrm{~km}\end{array}$ \\
\hline
\end{tabular}

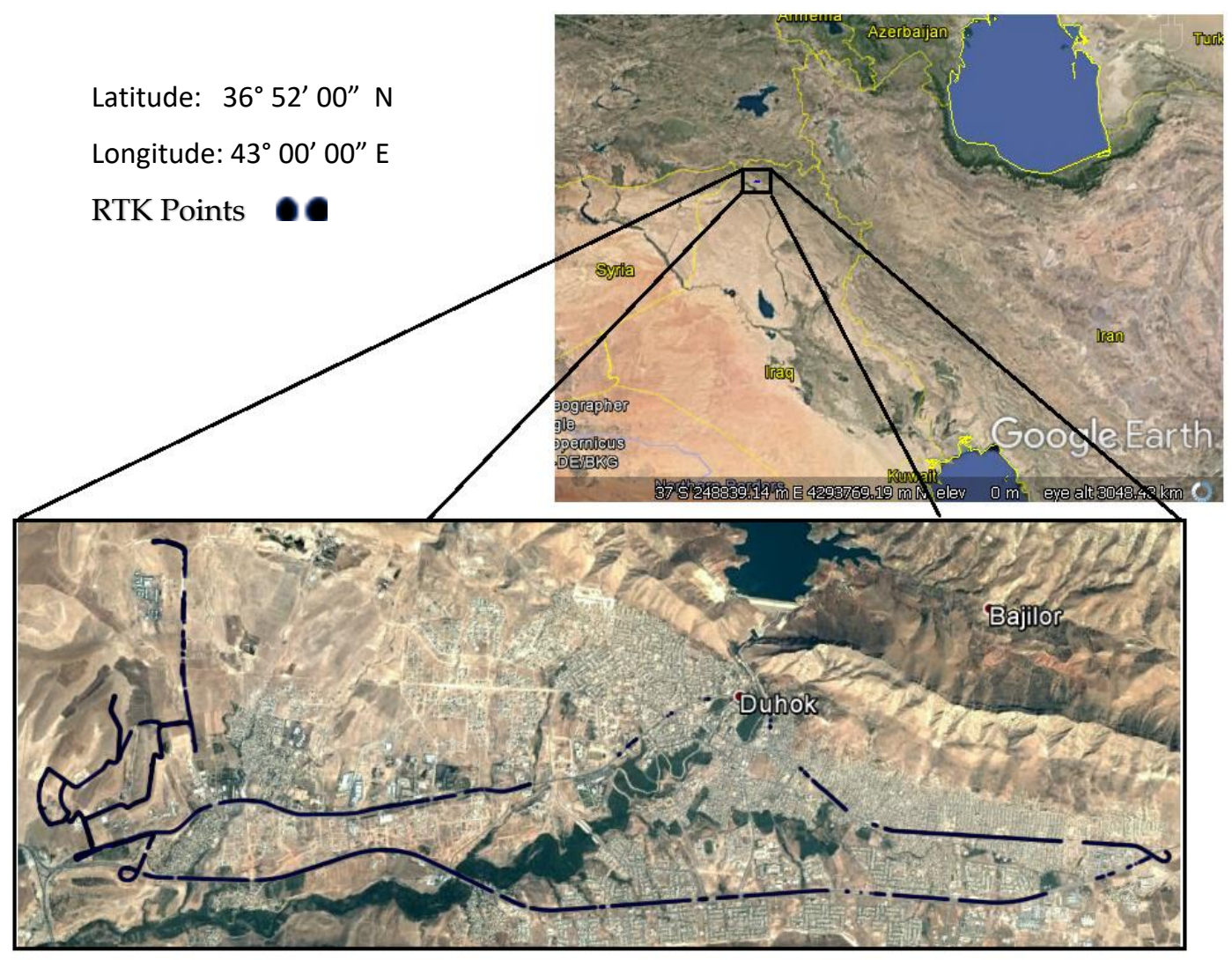

Fig (1) : Location and RTK data for the tested area

As discussed before, the first step in the data preparation took place with manually drawing the paths on the Google Earth imagery. The path was drawn manually and visually using the centerline of the road as illustrated in Figure 2. It is worth mentioning that the user is able to employ the road network data drawn by the company itself. However, for a better comparison, the authors decided to utilize their own extracted data. 


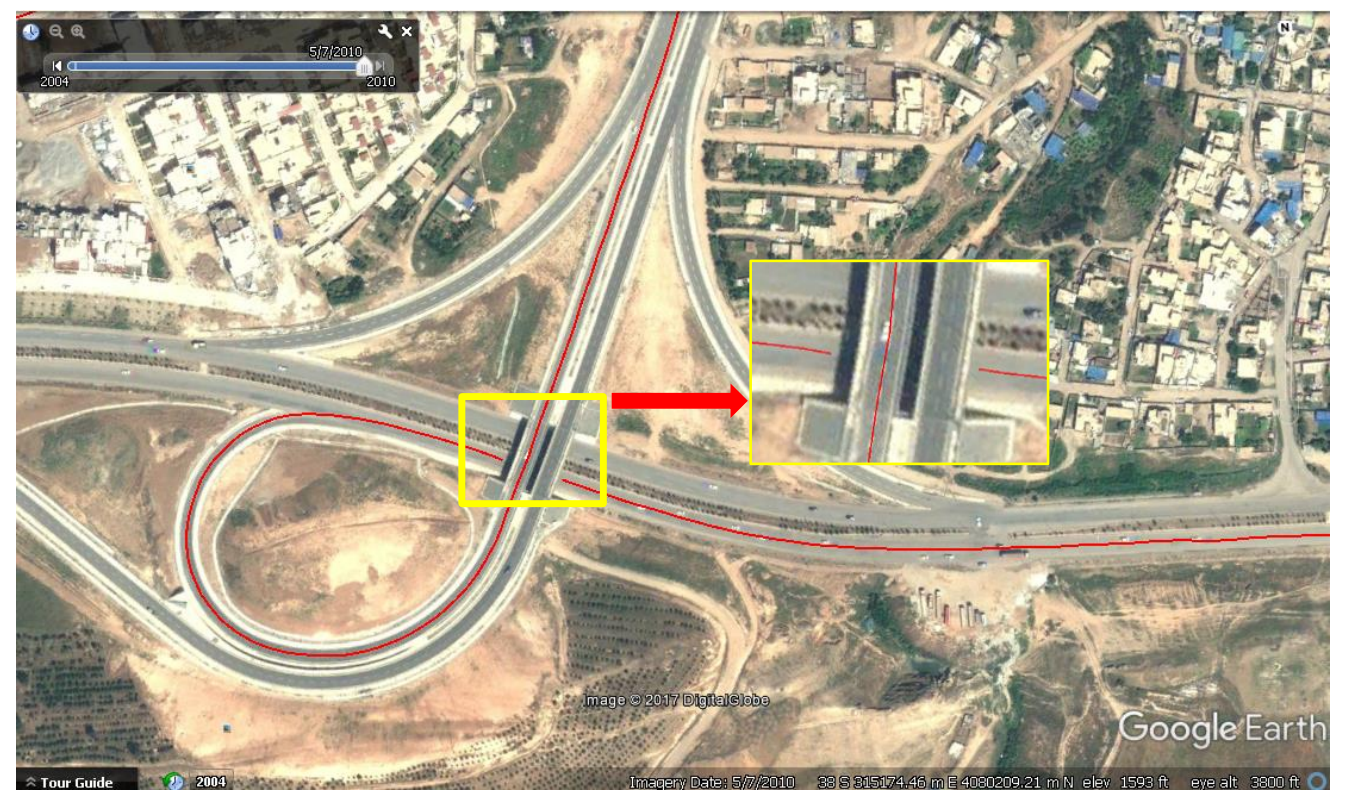

Fig (2) : Manually extracted centerline path using Google imagery 2010

At this stage, Google path was extracted with about 38 $\mathrm{km}$ length in total. Although there might be some places that the imagery has low resolution, the path was drawn as accurately as possible. The data from both sets (Google \& RTK) are input for the other stage which involves the processes and calculations of the RMSE and other related statistics in the fully automated sense.

\subsection{The workflow of the algorithm}

After extracting the road centerline, the next stage of data processing will start by automatically calculate the differences between the RTK and imagery road centerline. For this purpose, a Matlab algorithm was developed, Figure 3, to automatically calculate the variances between the manually created centerline and RTK points. First, the user has to input two sets of data to the subroutine. The first set of data is RTK points and the second is the centerline of the road was manually drawn as vertices or points for any imagery sources using CAD software or any equivalent tool. The centerline which is represented by a polyline, is divided using CAD into a number of points with a distance between every two points set by the user. From every RTK point, the algorithm works by searching for the nearest two points on the centerline. Then, it calculates the minimum distance (error) \& direction between the RTK point and the segment of the two nearest points. Now, for each point in RTK, there is a vector (distance with direction) which represents the error between the RTK and the centerline. The mean and RMS of errors are then determined. Then errors can be drawn as residuals to show clearly the trend of variation between the two datasets. 


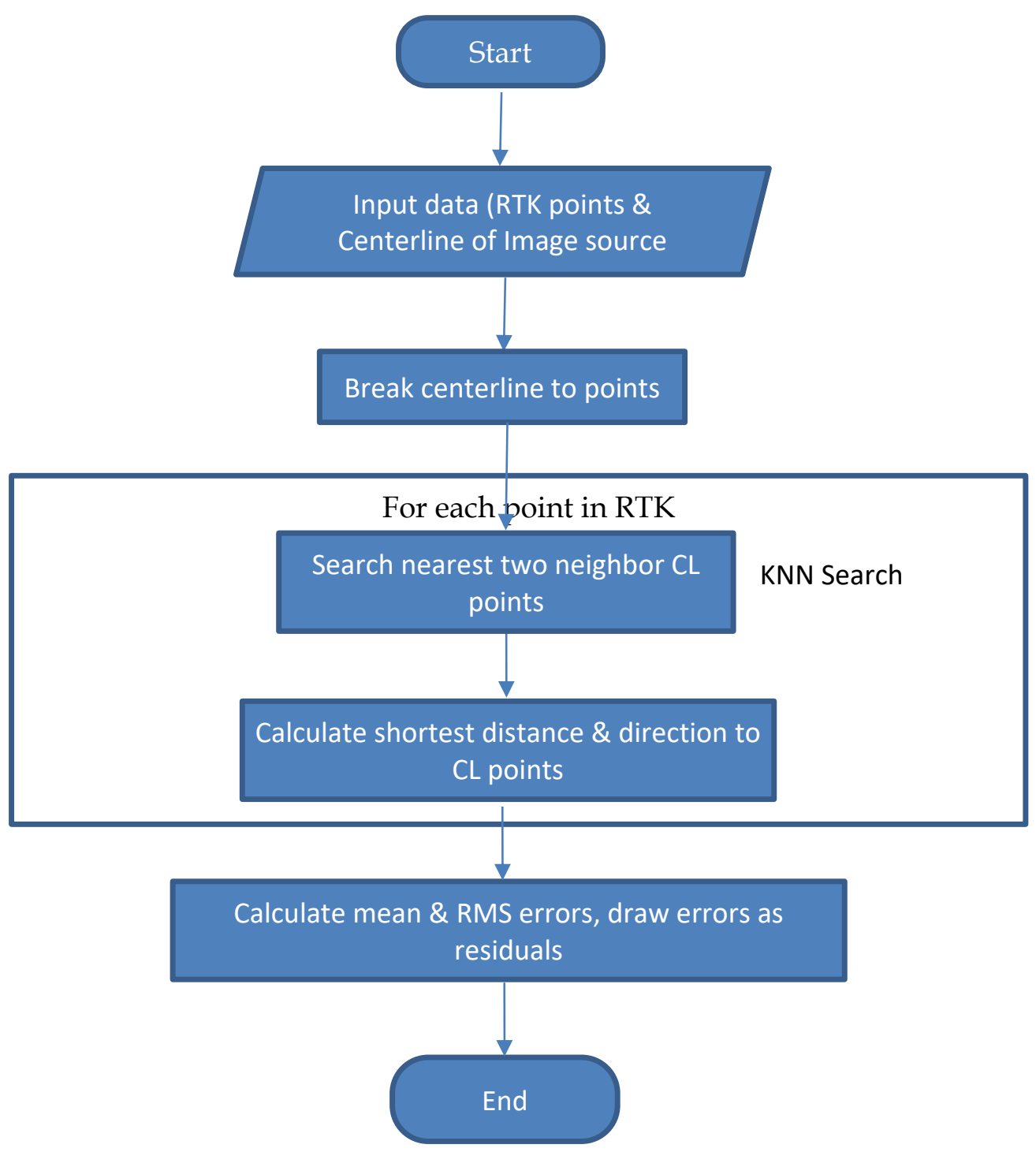

Fig (3) : Flowchart showing the important process in algorithm

\section{Research Related Formulas}

Below is the list of the formulas used in the algorithm to automatically calculate the differences between both sets of input data (RTK and Google road centerline points).

The CAD tool was used to divide Google polyline into equal segments and return points as shown in figure 4 .

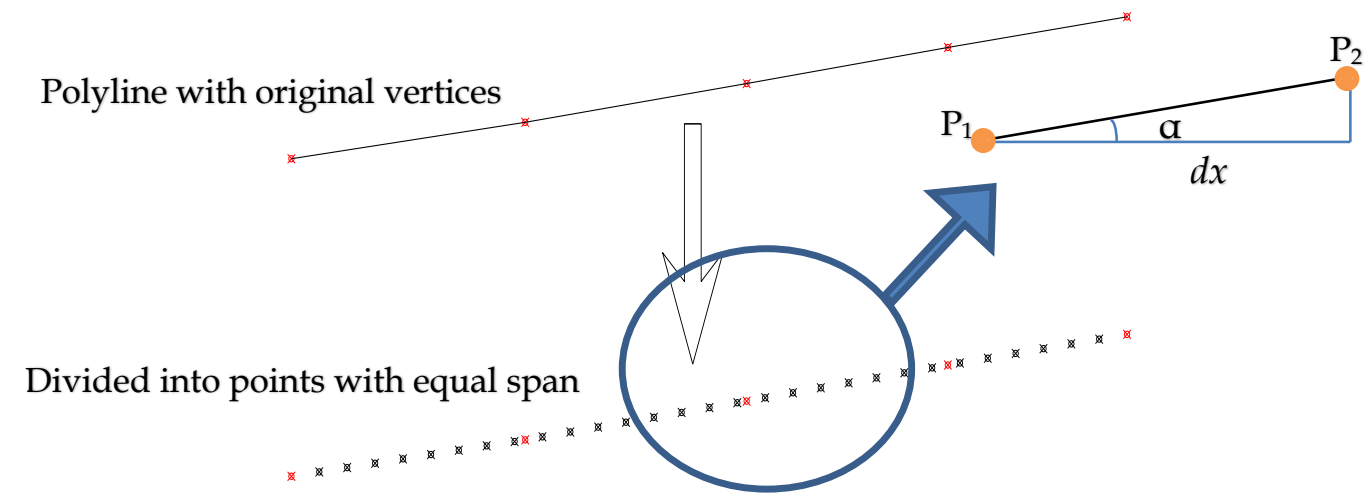

Fig (4) : Division of Google center line into points with equal spans 
$\alpha=\tan ^{-1} \frac{d y}{d x}$

$x_{n}=x_{1}+d x_{n}$

$y_{n}=y_{1}+d y_{n}$

Where:

$\alpha$ : Angle describing direction of P1-P2

$d y=(y 1-y 2) ; d x=(x 1-x 2)$

$x_{n}, y_{n}$ : New points

$d x_{n}$ : Distance between points along $\mathrm{x}$

$d y_{n}$ : Distance between points along y

1. KNN (K-Nearest Neighbors) search was used to find two neighboring points to an RTK point. The KNN search algorithm on Matlab was utilized with Euclidean distance as a method.

$D=\sqrt{d x^{2}+d y^{2}}$

2. The shortest distance between the RTK points and the segment of the two closest points was calculated as illustrated in figure 5 .

$E=\frac{\left|\left(a x_{0}+b y_{0}+c\right)\right|}{\left.\sqrt{a^{2}+b^{2}}\right)}$

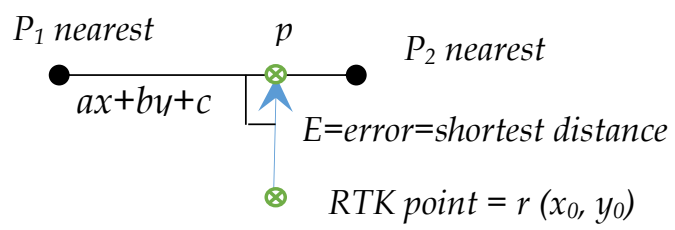

$A Z_{r p}=A Z_{p 1 p 2} \pm\left[\begin{array}{c}90 \\ 270\end{array}\right] \quad$ Quarter dependent

3. The Mean and RMS errors.
Mean Error $=\frac{\sum \text { errors }}{n}$

$R M S E=\sqrt{\frac{\sum \text { errors }^{2}}{n}}$

4. Results and Analysis

The output from the algorithm is an array of errors with the length equal to the number of RTK points that has been used in the process. Along with each error, the algorithm calculates the direction of error with reference to north meridian. Within seconds, many important statistical analysis can be worked out from the output information. The maximum error, Mean error, RMSE, and normal distribution curve were calculated from the output data. For the Google imagery source within the tested area, the maximum error was found to be $7.76 \mathrm{~m}$. The RMS errors was also calculated and found to be $1.53 \mathrm{~m}$, whereas, mean of errors was found to be $1.23 \mathrm{~m}$. The RMSE on this study was found to be close to the results published by Nagi Zomrawi Mohammed et al. (2013) and Farah and Algarni (2014). The array of differences can be statistically tested to see if the data was normally distributed, as shown in Figure 6. The differences were normally distributed with some values far away from the mean value. For the errors of more than $4 \mathrm{~m}$, the probability of density function was at the minimum range. Indicating that almost all the differences were less than the declared value. As normal distribution curve shows, all the differences were worked out as absolute value. The direction of error was considered in this study and no attempt was made to assign the positive or negative sign to the errors.

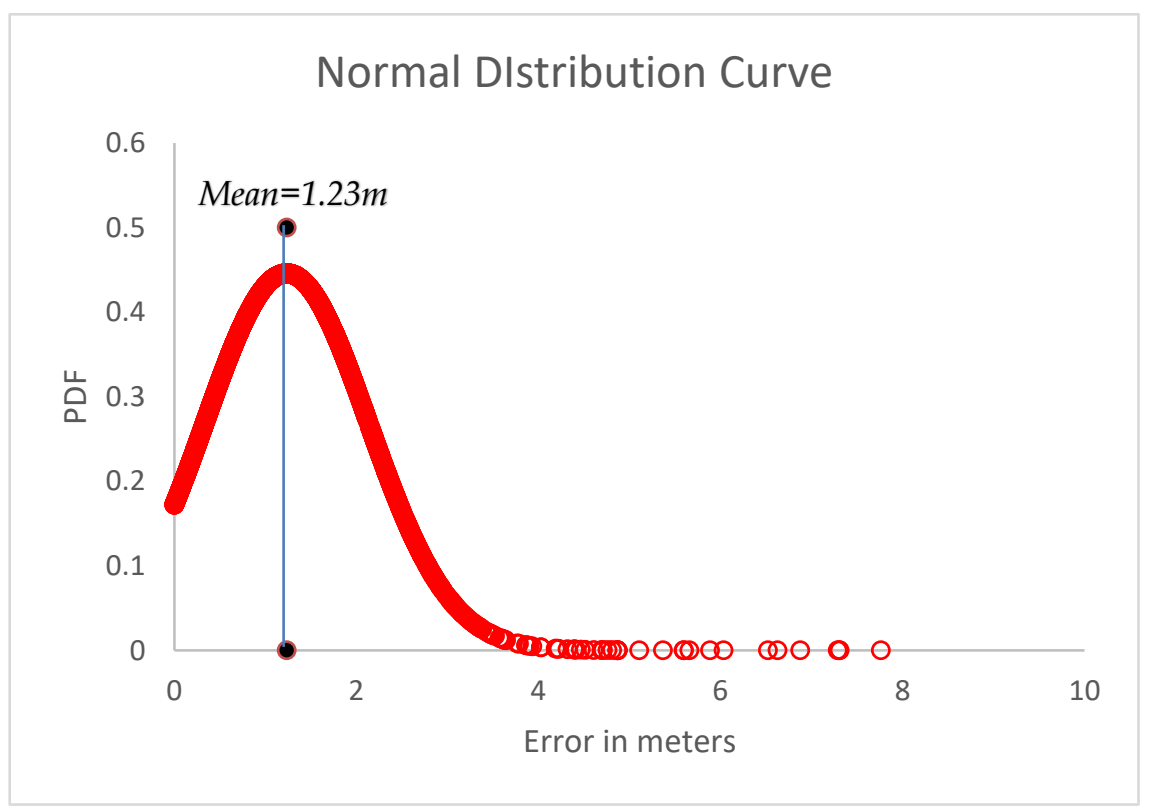

Fig (5) : Gaussian normal distribution of differences

As discussed previously, the algorithm worked out the direction (azimuth) of error. The errors with their directions can be drawn as a residuals, see figure 7 . Drawing the residuals will visually help to check 
whether the image source has any constant or any other trend shift relevant to the RTK points.

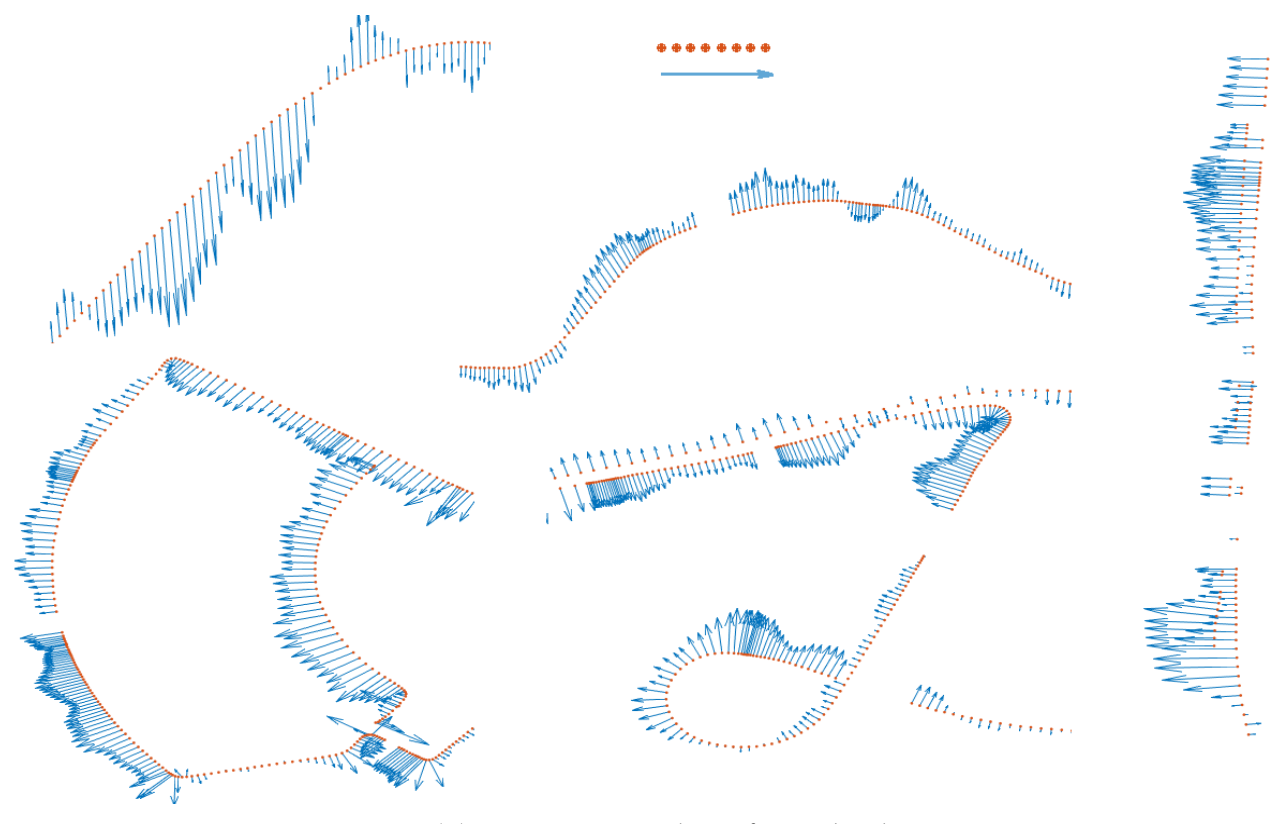

Fig (6) : Some samples of residuals

As figure 7 shows, the residuals indicate that the Google earth imagery has shifted towards the west direction. For the road located on the north-south direction, the shift was noticed to always be in the west direction. For other road alignment, for example the west-east direction, it can be noticed that the residuals were located in both directions. This indicates that the Google imagery might be stretched along the east-west direction.

\section{Conclusion}

This study has presented a new idea of calculating the error in the Google Earth imagery source using the semi-automated method. This method has some advantages over other research methods listed in the introductory section, such as saving the time of processing. It also has the advantage of including many points in the processing. For most scholars, the data collection was limited in a number of points. This was overcome by developing an algorithm to calculate the differences automatically. This method can be used in many other imagery sources in addition to the Google Earth. As long as the user has collected the road center via RTK mode, it can be applied to an unlimited number of image services. However, this approach can only be carried out in flat areas or in areas where mobile platforms can be accessed in order to collect the center line of the road using RTK GPS.

Future work will include the trials toward fully automated method. The centerline road extraction can be done in fully automated bases without an intervention. In the near future, it is better to include other imagery sources in the process and analysis using the developed method.

\section{References}

1. EL-HALLAQ, M. A. \& HAMAD, M. I. (2017). Positional Accuracy of the Google Earth Imagery In The Gaza Strip. Journal of Multidisciplinary Engineering Science and Technology (JMEST), JMESTN42352184, Vol. 4

2. FARAH, A. \& ALGARNI, D. (2014). Positional Accuracy Assessment of Google Earth in Riyadh. Artficial Satellites, 49, 102-106.

3. KAZIMIERZ, B., KHAIRUNNISA, I. \& BRUNEI, D. (2011). On the Positional Accuracy of the Google Earth Imagery. Bridging the Gap between Cultures Marrakech, Morocco.

4. LEACHTENAUER, J. C., DANIEL, K. \& VOGL, T. P. (1997). Digitizing Corona imagery: Quality vs. cost. In Corona: Between the Sun \& the Earth, The first NRO reconnaissance eye in space. American Society Photogrammetry and Remote Sensing.

5. MISSIONS, S. (2018). WorldView-2 "Earth Observation Resources" [Online]. online: eoPortal Dictionary. Available: https://directory.eoportal.org/web/eoportal/satellitemissions/v-w-x-y-z/worldview-2 [Accessed 2 Febrauary 2018].

6. NAGI ZOMRAWI MOHAMMED, AHMED GHAZI \& MUSTAFA., H. E. (2013). Positional Accuracy Testing of Google Earth. . International Journal of Multidisciplinary Sciences and Engineering, 4, 6-9.

7. NIIRS. (1996). Imagery Resolution Assessment and 
Reporting Standards Committee. Civil National Imagery Interpretability Rating Scale (NIIRS) [Online]. Available: www.fas.org/irp/imint/niirs_c/index.html [Accessed 11 November 2017].

8. PAREDES-HERNANDEZ, C., SALINASCASTILLO, W., GUEVARA-CORTINA, F. \& MARTINEZ-BECERRA, X. (2013). Horizontal Positional Accuracy of Google Earth's imagery Over Rural Areas: A Study Case in Tamaulipas, Mexico. BCG - Boletim de Ciências Geodésicas - On-Line version 19.

9. POTERE, D. (2008). Horizontal Positional Accuracy of Google Earth's HighResolution Imagery Archive. Sensors, 8, 7973-7981.

10. RAGHEB, A. E. \& RAGAB, A. F. (2015). Enhancement of Google Earth Positional Accuracy. International Journal of Engineering Research \& Technology (IJERT), IJERTV4IS010732, 4.

11. UBUKAWA, T. (2013). An Evaluation of the Horizontal Positional Accuracy of Google and Bing Satellite Imagery and Three Roads Data Sets Based on High Resolution Satellite Imagery. Center for International Earth Science Information Network (CIESIN). The Earth Institute at Columbia University. 